\title{
Optical implementations of two-dimensional fractional Fourier transforms and linear canonical transforms with arbitrary parameters
}

\author{
Aysegul Sahin, Haldun M. Ozaktas, and David Mendlovic
}

\begin{abstract}
We provide a general treatment of optical two-dimensional fractional Fourier transforming systems. We not only allow the fractional Fourier transform orders to be specified independently for the two dimensions but also allow the input and output scale parameters and the residual spherical phase factors to be controlled. We further discuss systems that do not allow all these parameters to be controlled at the same time but are simpler and employ a fewer number of lenses. The variety of systems discussed and the design equations provided should be useful in practical applications for which an optical fractional Fourier transforming stage is to be employed. (C) 1998 Optical Society of America
\end{abstract}

OCIS code: 070.2590 .

\section{Introduction}

The fractional Fourier transform has received considerable attention since 1993. ${ }^{1-13}$ Several applications of the fractional Fourier transform have been suggested. In particular, many signal- and imageprocessing applications have been developed on the basis of the fractional Fourier transform. ${ }^{14-25}$ Several two-dimensional (2-D) optical implementations have been discussed previously, $1,4,6,8,11,26-28$ but a comprehensive and systematic treatment did not exist until Ref. 29, in which we provided a detailed examination of the 2-D fractional Fourier transform.

In this paper we provide a very general treatment of optical 2-D fractional Fourier transforming systems. We allow the fractional Fourier transform orders to be specified independently for the two dimensions. We also allow the input and output scale parameters and the residual spherical phase factors to be controlled. We further discuss systems that do not allow all of these parameters to be controlled at the same time but are simpler and employ a fewer number of lenses.

When this research was performed, A. Sahin and H. M. Ozaktas were with the Department of Electrical Engineering, Bilkent University, TR-06533 Bilkent, Ankara, Turkey; D. Mendlovic was with the Faculty of Engineering, Tel-Aviv University, 69978 Tel-Aviv, Israel. A. Sahin is now with the Department of Economics, University of Rochester, Rochester, New York 14627.

Received 17 June 1997; revised manuscript received 4 December 1997.

0003-6935/98/112130- $12 \$ 15.00 / 0$

(C) 1998 Optical Society of America
We begin by reviewing the properties of the 2-D fractional Fourier transform. Some of these are trivial extensions of the corresponding onedimensional (1-D) property or have been discussed elsewhere, although they do not appear collectively in any single source. For this reason we list them with minimum comment.

In Section 3 we present optical realizations of linear canonical transforms. Since linear canonical transforms can be interpreted as scaled fractional Fourier transforms with additional phase terms, these systems can realize fractional Fourier transforms with desired orders, scale factors, and residual phase terms. In Section 4 we consecutively consider systems with two, four, and six cylindrical lenses. In each case we discuss which parameters it is possible to specify independently and which parameters we have no control over. One can choose from among these systems the one that provides the required flexibility with the minimum number of lenses.

\section{Two-Dimensional Fractional Fourier Transform}

The 2-D fractional Fourier transform with the orders $a_{x}$ for the $x$ axis and $a_{y}$ for the $y$ axis, for $0<\left|a_{x}\right|<2$ and $0<\left|a_{y}\right|<2$, respectively, is defined as

$$
\begin{aligned}
\mathscr{F}^{a_{x}, a_{y}}[f(x, y)](x, y)= & \int_{-\infty}^{\infty} \int_{-\infty}^{\infty} B_{a_{x}, a_{y}}\left(x, y ; x^{\prime}, y^{\prime}\right) \\
& \times f\left(x^{\prime}, y^{\prime}\right) \mathrm{d} x^{\prime} \mathrm{d} y^{\prime},
\end{aligned}
$$


where

$$
\begin{aligned}
B_{a_{x}, a_{y}}\left(x, y ; x^{\prime}, y^{\prime}\right)= & B_{a_{x}}\left(x, x^{\prime}\right) B_{a_{y}}\left(y, y^{\prime}\right), \\
B_{a_{x}}\left(x, x^{\prime}\right)= & A_{\phi_{x}} \exp \left[i \pi \left(x^{2} \cot \phi_{x}\right.\right. \\
& \left.\left.-2 x x^{\prime} \csc \phi_{x}+x^{\prime 2} \cot \phi_{x}\right)\right], \\
B_{a_{y}}\left(y, y^{\prime}\right)= & A_{\phi_{y}} \exp \left[i \pi \left(y^{2} \cot \phi_{y}\right.\right. \\
& \left.\left.-2 y y^{\prime} \csc \phi_{y}+y^{\prime 2} \cot \phi_{y}\right)\right], \\
A_{\phi_{x}}= & \frac{\exp \left[-i\left(\pi \hat{\phi}_{x} / 4-\phi_{x} / 2\right)\right]}{\left(\left|\sin \phi_{x}\right|\right)^{1 / 2}} \\
A_{\phi_{y}}= & \frac{\exp \left[-i\left(\pi \hat{\phi}_{y} / 4-\phi_{y} / 2\right)\right]}{\left(\left|\sin \phi_{y}\right|\right)^{1 / 2}},
\end{aligned}
$$

$\phi_{x}=a_{x} \pi / 2, \phi_{y}=a_{y} \pi / 2, \hat{\phi}_{x}=\operatorname{sgn}\left(\phi_{x}\right)$, and $\hat{\phi}_{y}=$ $\operatorname{sgn}\left(\phi_{y}\right)$. As Eq. (2) suggests, the kernel $B_{a_{x}, a_{y}}$ is a separable kernel.

The definition may be simplified by use of vectormatrix notation:

$$
\begin{aligned}
\mathscr{F}[f(\mathbf{r})](\mathbf{r})= & \int_{-\infty}^{\infty} A_{\phi_{\mathbf{r}}} \exp \left[i \pi \left(\mathbf{r}^{T} \mathbf{C}_{\mathbf{t}} \mathbf{r}-2 \mathbf{r}^{T} \mathbf{C}_{\mathbf{s}} \mathbf{r}^{\prime}\right.\right. \\
& \left.\left.+\mathbf{r}^{\prime T} \mathbf{C}_{\mathbf{t}} \mathbf{r}^{\prime}\right)\right] f\left(\mathbf{r}^{\prime}\right) \mathrm{d} \mathbf{r}^{\prime}
\end{aligned}
$$

where

$$
\begin{aligned}
A_{\phi_{\mathbf{r}}} & =A_{\phi_{x}} A_{\phi_{y}}, \quad \mathbf{r}=\left[\begin{array}{ll}
x & y
\end{array}\right]^{T}, \quad \mathbf{r}^{\prime}=\left[\begin{array}{ll}
x^{\prime} & y^{\prime}
\end{array}\right]^{T}, \\
\mathbf{C}_{\mathbf{t}} & =\left[\begin{array}{cc}
\cot \phi_{x} & 0 \\
0 & \cot \phi_{y}
\end{array}\right], \quad \mathbf{C}_{\mathbf{s}}=\left[\begin{array}{cc}
\csc \phi_{x} & 0 \\
0 & \csc \phi_{y}
\end{array}\right] .
\end{aligned}
$$

Two- (and higher-) dimensional ${ }^{30}$ fractional Fourier transforms were first considered with equal orders $a_{x}=a_{y}$, and their optical implementations involved spherical lenses and graded-index media (see the references in the first paragraph of Section 1). The possibility of different orders was mentioned in Ref. 4 and discussed in Refs. 26-28.

\section{A. Properties of Two-Dimensional Fractional Fourier Transforms}

Most of the following properties are straightforward generalizations of the $1-D$ versions. ${ }^{31-33}$

\section{Additivity}

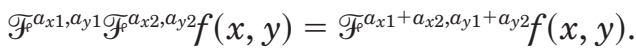

\section{Linearity}

For arbitrary constants $c_{k}$ we find

$$
\mathscr{F}^{a_{x}, a_{y}} \sum_{k} c_{k} f(x, y)=\sum_{k} c_{k} \mathscr{F}^{a_{x}, a_{y}} f(x, y) .
$$

\section{Separability}

If $f(x, y)=f(x) f(y)$, then

$$
\mathscr{F}^{a_{x}, a_{y}} f(x, y)=\left[\mathscr{F}^{a_{x}} f(x)\right]\left[\mathscr{F}^{a_{y}} f(y)\right] .
$$

4. Inverse Transform

$$
B_{a_{x}, a_{y}}{ }^{-1}\left(x, y ; x^{\prime}, y^{\prime}\right)=B_{-a_{x},-a_{y}}\left(x, y ; x^{\prime}, y^{\prime}\right) .
$$

\section{Unitarity}

The 2-D kernel is unitary, as shown by

$$
\begin{aligned}
B_{a_{x}, a_{y}}^{-1}\left(x^{\prime}, y^{\prime} ; x, y\right) & =B_{-a_{x},-a_{y}}\left(x^{\prime}, y^{\prime} ; x, y\right) \\
& =B_{a_{x}, a_{y}}^{*}\left(x, y ; x^{\prime}, y^{\prime}\right),
\end{aligned}
$$

where the asterisk denotes the complex conjugate.

\section{Parseval Relation}

$$
\begin{aligned}
\int_{-\infty}^{\infty} \int_{-\infty}^{\infty} f\left(\mathbf{r}^{\prime}\right)^{*} g\left(\mathbf{r}^{\prime}\right) \mathrm{d} \mathbf{r}^{\prime} & =\int_{-\infty}^{\infty} \int_{-\infty}^{\infty}\left\{\mathscr{F}^{a_{x}, a_{y}} f\left(\mathbf{r}^{\prime}\right)\right\}^{*} \\
& \times\left\{\mathscr{F}^{a_{x}, a_{y}} g\left(\mathbf{r}^{\prime}\right)\right\} \mathrm{d} \mathbf{r}^{\prime}, \\
\int_{-\infty}^{\infty} \int_{-\infty}^{\infty}\left|f\left(\mathbf{r}^{\prime}\right)\right|^{2} \mathrm{~d} \mathbf{r}^{\prime}= & \int_{-\infty}^{\infty} \int_{-\infty}^{\infty}\left|\mathscr{F}^{a_{x}, a_{y}} f\left(\mathbf{r}^{\prime}\right)\right|^{2} \mathrm{~d} \mathbf{r}^{\prime} .
\end{aligned}
$$

\section{Effect of the Coordinate Shift}

The fractional Fourier transform of $f\left(x-x_{0}, y-y_{0}\right)$ can be expressed in terms of the fractional Fourier transform of $f(x, y)$ as

$$
\begin{aligned}
\mathscr{F}^{a_{x}, a_{y}}\left[f\left(\mathbf{r}-\mathbf{r}_{0}\right)\right](\mathbf{r})= & \exp \left\{-i 2 \pi\left[\mathbf{r}_{\mathbf{s}}^{T}\left(\mathbf{r}-\frac{1}{2} \mathbf{r}_{\mathbf{c}}\right)\right]\right\} \\
& \times \mathscr{F}^{a_{x}, a_{y}}[f(\mathbf{r})]\left(\mathbf{r}-\mathbf{r}_{\mathbf{c}}\right),
\end{aligned}
$$

where

$$
\begin{gathered}
\mathbf{r}_{0}=\left[\begin{array}{ll}
x_{0} & y_{0}
\end{array}\right]^{T}, \quad \mathbf{r}_{\mathbf{c}}=\left[\begin{array}{ll}
x_{0} \cos \phi_{x} & y_{0} \cos \phi_{y}
\end{array}\right]^{T}, \\
\mathbf{r}_{\mathbf{s}}=\left[\begin{array}{ll}
x_{0} \sin \phi_{x} & y_{0} \sin \phi_{y}
\end{array}\right]^{T} .
\end{gathered}
$$

\section{Effect of Multiplication by a Complex Exponential}

If a function $f(x, y)$ is multiplied by an exponential $\exp \left[i 2 \pi\left(m_{x} x+m_{y} y\right)\right]$, the resulting fractional Fourier transform becomes

$$
\begin{aligned}
\mathscr{F}^{a_{x}, a_{y}}\left[\exp \left(i 2 \pi \mathbf{m}^{T} \mathbf{r}\right) f(\mathbf{r})\right]= & \exp \left\{i \pi\left[\mathbf{m}_{\mathbf{c}}{ }^{T}\left(\mathbf{m}_{\mathbf{s}}+2 \mathbf{r}\right)\right]\right\} \\
& \times \mathscr{F}^{a_{x}, a_{y}}[f(\mathbf{r})]\left(\mathbf{r}-\mathbf{m}_{\mathbf{s}}\right),
\end{aligned}
$$

where

$$
\begin{gathered}
\mathbf{m}=\left[\begin{array}{ll}
m_{x} & m_{y}
\end{array}\right]^{T}, \quad \mathbf{c}=\left[\begin{array}{ll}
m_{x} \cos \phi_{x} & m_{y} \cos \phi_{y}
\end{array}\right]^{T}, \\
\mathbf{m}_{\mathbf{s}}=\left[\begin{array}{ll}
m_{x} \sin \phi_{x} & m_{y} \sin \phi_{y}
\end{array}\right]^{T} .
\end{gathered}
$$




\section{Multiplication by Powers of the Coordinate Variables}

The fractional Fourier transform of $x^{m} y^{n} f(x, y)$ for $m$, $n \geq 0$ is

$$
\begin{aligned}
\mathscr{F}^{a_{x}, a_{y}}\left[x^{m} y^{n} f(x, y)\right]= & \left(x \cos \phi_{x}+\frac{i}{\pi} \sin \phi_{x} \frac{\partial}{\partial x}\right)^{m} \\
& \times\left(y \cos \phi_{y}+\frac{i}{\pi} \sin \phi_{y} \frac{\partial}{\partial y}\right)^{n} \\
& \times \mathscr{F}^{a_{x}, a_{y}} f(x, y) .
\end{aligned}
$$

10. Derivative of $f(x, y)$

The fractional Fourier transform of the term $\left(\partial^{m} / \partial^{m} x\right)\left(\partial^{n} / \partial^{n} y\right) f(x, y)$ is

$$
\begin{aligned}
\mathscr{F}^{a_{x}, a_{y}}\left[\frac{\partial^{m}}{\partial x^{m}} \frac{\partial^{n}}{\partial y^{n}} f(x, y)\right]= & \left(i 2 \pi x \sin \phi_{x}+\cos \phi_{x} \frac{\partial}{\partial x}\right)^{m} \\
& \times\left(i 2 \pi y \sin \phi_{y}+\cos \phi_{y} \frac{\partial}{\partial y}\right)^{n} \\
& \times \mathscr{F}^{a_{x}, a_{y}} f(x, y) .
\end{aligned}
$$

\section{Scaling}

The fractional Fourier transform of $f\left(k_{x} x, k_{y} y\right)$ can be represented in terms of the fractional Fourier transform of $f(x, y)$ with different orders $a_{x}{ }^{\prime}$ and $a_{y}{ }^{\prime}$ as

$$
\mathscr{F}^{a_{x}, a_{y}}[f(\mathbf{K r})](\mathbf{r})=C \exp \left(i \pi \mathbf{r}^{T} \mathbf{D r}\right) \mathscr{F}^{a_{x}^{\prime}, a_{y}^{\prime}}[f(\mathbf{r})]\left(\mathbf{K}^{\prime} \mathbf{r}\right),
$$

where

$$
\begin{aligned}
& C=\frac{A_{\phi_{x}} A_{\phi_{y}}}{\left|k_{x}\right|\left|k_{y}\right| A_{\phi_{x}} A_{\phi_{y}}}, \\
& \mathbf{K}=\left[\begin{array}{cc}
k_{x} & 0 \\
0 & k_{y}
\end{array}\right] \text {, } \\
& \phi_{x}{ }^{\prime}=\arctan \left(k_{x}{ }^{2} \tan \phi_{x}\right), \quad a_{x}{ }^{\prime}=\frac{2 a_{\phi_{x}{ }^{\prime}}}{\pi}, \\
& \phi_{y}{ }^{\prime}=\arctan \left(k_{y}{ }^{2} \tan \phi_{y}\right), \quad a_{y}{ }^{\prime}=\frac{2 a_{\phi_{y}{ }^{\prime}}}{\pi}, \\
& \mathbf{D}=\left[\begin{array}{cc}
\cot \phi_{x} \frac{k_{x}{ }^{4}-1}{k_{x}{ }^{4}+\cot ^{2} \phi_{x}} & 0 \\
0 & \cot \phi_{y} \frac{k_{y}{ }^{4}-1}{k_{y}{ }^{4}+\cot ^{2} \phi_{y}}
\end{array}\right], \\
& \mathbf{K}^{\prime}=\left[\begin{array}{cc}
\frac{\sin \phi_{x}{ }^{\prime}}{k_{x} \sin \phi_{x}} & 0 \\
0 & \frac{\sin \phi_{y}{ }^{\prime}}{k_{y} \sin \phi_{y}}
\end{array}\right] .
\end{aligned}
$$

\section{Rotation}

Let

$$
\mathbf{R}=\left[\begin{array}{cc}
\cos \theta & \sin \theta \\
-\sin \theta & \cos \theta
\end{array}\right]
$$

then $f(\mathbf{R r})=f(\cos \theta x+\sin \theta y,-\sin \theta x+\cos \theta y)$ represents the rotated function with the angle $\theta$. When it is the case that $a_{x}=a_{y}=a$, then

$$
\mathscr{F}^{a}[f(\mathbf{R r})](\mathbf{r})=\mathscr{F}^{a}(\mathbf{r})(\mathbf{R r}) .
$$

\section{Wigner Distribution and Fractional Fourier Transform}

Let $W_{f}\left(x, y ; v_{x}, v_{y}\right)$ be the Wigner distribution of $f(x, y)$. If $g(x, y)$ is the fractional Fourier transform of $f(x, y)$, then the Wigner distribution of $g(x, y)$ is related to that of $f(x, y)$ through the following:

$$
W_{g}(\mathbf{r}, \boldsymbol{v})=W_{f}(\mathbf{A r}+\mathbf{B} \boldsymbol{v}, \mathbf{C r}+\mathbf{D} \boldsymbol{v}),
$$

where

$$
\begin{aligned}
& \mathbf{r}=\left[\begin{array}{ll}
x & y
\end{array}\right]^{T,}, \boldsymbol{v}=\left[\begin{array}{ll}
v_{x} & v_{y}
\end{array}\right]^{T}, \\
& \mathbf{A}=\left[\begin{array}{cc}
\cos \phi_{x} & 0 \\
0 & \cos \phi_{y}
\end{array}\right], \quad \mathbf{B}=\left[\begin{array}{cc}
-\sin \phi_{x} & 0 \\
0 & -\sin \phi_{y}
\end{array}\right], \\
& \mathbf{C}=\left[\begin{array}{cc}
\sin \phi_{x} & 0 \\
0 & \sin \phi_{y}
\end{array}\right], \quad \mathbf{D}=\left[\begin{array}{cc}
\cos \phi_{x} & 0 \\
0 & \cos \phi_{y}
\end{array}\right] .
\end{aligned}
$$

As Eq. (20) suggests, the effect of the fractional Fourier transform on the Wigner distribution is a counterclockwise rotation with the angle $\phi_{x}$ in the $x-v_{x}$ plane and the angle $\phi_{y}$ in the $y-v_{y}$ plane.

\section{Projection}

The projection property of a 1-D kernel ${ }^{34}$ states that the projection of the Wigner distribution function on an axis making an angle $\phi$ with the $x$ axis is the absolute square of the fractional Fourier transform of the function with the order $a(\phi=a \pi / 2)$. This effect can be represented in terms of the Radon transform as

$$
\mathscr{R}_{\phi}[W(x, v)]=\left|\mathscr{F}^{a}[f(x)]\right|^{2},
$$

where the Radon transform of a 2-D function is its projection on an axis making an angle $\phi$ with the $x$ axis. The separability of the 2-D kernel can be used to derive the corresponding property for the 2-D case. If the Radon transform is applied successively to the Wigner distribution $W\left(x, y ; v_{x}, v_{y}\right)$, then the property becomes

$$
\mathscr{R}_{\phi_{y}}\left\{\mathscr{R}_{\phi_{x}}\left[W\left(x, y ; v_{x}, v_{y}\right)\right]\right\}=\left|\mathscr{F}^{a_{x}, a_{y}}[f(x, y)]\right|^{2} .
$$

Thus the projection of the Wigner distribution $W(x, y$; $\left.v_{x}, v_{y}\right)$ of any function $f(x, y)$ on the plane determined by two lines-the first making an angle $\phi_{x}$ with the $x$ axis and the second making an angle $\phi_{y}$ with the $y$ axis-is the absolute square of its 2-D fractional Fourier transform with the orders $a_{x}$ and $a_{y}$. 


\section{Eigenvalues and Eigenfunctions}

Two-dimensional Hermite-Gaussian functions are eigenfunctions of the 2-D fractional Fourier transform:

$$
\begin{array}{r}
\int_{-\infty}^{\infty} \int_{-\infty}^{\infty} B_{a_{x}, a_{y}}\left(x, y ; x^{\prime}, y^{\prime}\right) \Psi_{n m}\left(x, y^{\prime}\right) \mathrm{d} x^{\prime} \mathrm{d} y^{\prime} \\
=\lambda_{n m} \Psi_{n m}(x, y),
\end{array}
$$

where

$$
\begin{aligned}
\Psi_{n m}(x, y)= & \frac{2^{1 / 2}}{\left(2^{n} 2^{m} n ! m !\right)^{1 / 2}} H_{n}(\sqrt{2 \pi} x) H_{m}(\sqrt{2 \pi} y) \\
& \times \exp \left[-\pi\left(x^{2}+y^{2}\right)\right], \\
\lambda_{n m}= & \exp \left(-i \pi a_{x} n / 2\right) \exp \left(-i \pi a_{y} m / 2\right) .
\end{aligned}
$$

B. Linear Canonical Transforms and Fractional Fourier Transforms

Fractional Fourier transforms, Fresnel transforms, chirp multiplication, and scaling operations are used widely in optics to analyze systems composed of sections of free space and thin lenses. These linear integral transforms belong to the class of linear canonical transforms. The definition for a 2-D linear canonical transform is

$$
\begin{aligned}
g(x, y)= & \int_{-\infty}^{\infty} \int_{-\infty}^{\infty} h\left(x, y ; x^{\prime}, y^{\prime}\right) f\left(x^{\prime}, y^{\prime}\right) \mathrm{d} x^{\prime} \mathrm{d} y^{\prime}, \\
h\left(x, y ; x^{\prime}, y^{\prime}\right)= & \exp (-i \pi / 4) \beta_{x}{ }^{1 / 2} \\
& \times \exp \left[i \pi\left(\alpha_{x} x^{2}-2 \beta_{x} x x^{\prime}+\gamma_{x} x^{\prime 2}\right)\right] \\
& \times \exp (-i \pi / 4) \beta_{y}{ }^{1 / 2} \exp \left[i \pi \left(\alpha_{y} y^{2}\right.\right. \\
& \left.\left.-2 \beta_{y} y y^{\prime}+\gamma_{y} y^{\prime 2}\right)\right],
\end{aligned}
$$

where $\alpha_{x}, \beta_{x}, \gamma_{x}$ and $\alpha_{y}, \beta_{y}, \gamma_{y}$ are real constants. Any linear canonical transform is completely specified by its parameters. Alternatively, linear canonical transforms can be specified by use of a transformation matrix. The transformation matrix of such a system, as specified by the parameters $\alpha_{x}$, $\beta_{x}, \gamma_{x}$ and $\alpha_{y}, \beta_{y}, \gamma_{y}$, is

$$
\begin{aligned}
\mathbf{T} & \equiv\left[\begin{array}{cccc}
A_{x} & 0 & B_{x} & 0 \\
0 & A_{y} & 0 & B_{y} \\
C_{x} & 0 & D_{x} & 0 \\
0 & C_{y} & 0 & D_{y}
\end{array}\right] \\
& \equiv\left[\begin{array}{cccc}
\gamma_{x} / \beta_{x} & 0 & 1 / \beta_{x} & 0 \\
0 & \gamma_{y} / \beta_{y} & 0 & 1 / \beta_{y} \\
-\beta_{x}+\alpha_{x} \gamma_{x} / \beta_{x} & 0 & \alpha_{x} / \beta_{x} & 0 \\
0 & -\beta_{y}+\alpha_{y} \gamma_{y} / \beta_{y} & 0 & \alpha_{y} / \beta_{y}
\end{array}\right],
\end{aligned}
$$

with $A_{x} D_{x}-B_{x} C_{x}=1$ and $A_{y} D_{y}-B_{y} C_{y}=1 .^{35,36}$

Propagation in free space and through thin lenses can also be analyzed as special forms of linear canonical transforms. Here both the kernels and the transformation matrices of the optical components are given. The transformation kernel for free-space propagation of length $d$ is expressed as

$$
h_{f}\left(x, y, x^{\prime}, y^{\prime}\right)=K_{f} \exp \left\{i \pi\left[\frac{\left(x-x^{\prime}\right)^{2}}{\lambda d}+\frac{\left(y-y^{\prime}\right)^{2}}{\lambda d}\right]\right\} \text {, }
$$

and its corresponding transformation matrix is

$$
\mathbf{T}_{f}(d)=\left[\begin{array}{cccc}
1 & 0 & \lambda d & 0 \\
0 & 1 & 0 & \lambda d \\
0 & 0 & 1 & 0 \\
0 & 0 & 0 & 1
\end{array}\right] .
$$

Similarly, the kernel for a cylindrical lens with focal length $f_{x}$ along the $x$ direction is

$$
h_{x l}\left(x, y, x^{\prime}, y^{\prime}\right)=K_{x l} \delta\left(x-x^{\prime}\right) \exp \left(-i \pi x^{2} / \lambda f_{x}\right),
$$

with its transformation matrix given by

$$
\mathbf{T}_{x l}\left(f_{x}\right)=\left[\begin{array}{cccc}
1 & 0 & 0 & 0 \\
0 & 1 & 0 & 0 \\
\frac{-1}{\lambda f_{x}} & 0 & 1 & 0 \\
0 & 0 & 0 & 1
\end{array}\right],
$$

and the kernel for a cylindrical lens with a focal length $f_{y}$ along the $y$ direction is

$$
h_{y l}\left(x, y, x^{\prime}, y^{\prime}\right)=K_{y l} \delta\left(y-y^{\prime}\right) \exp \left(-i \pi y^{2} / \lambda f_{y}\right),
$$

with its transformation matrix given by

$$
\mathbf{T}_{y l}\left(f_{y}\right)=\left[\begin{array}{cccc}
1 & 0 & 0 & 0 \\
0 & 1 & 0 & 0 \\
0 & 0 & 1 & 0 \\
0 & \frac{-1}{\lambda f_{y}} & 0 & 1
\end{array}\right] .
$$

More general anamorphic lenses may be represented by a kernel of the form

$$
\begin{aligned}
h_{x y l}\left(x, y, x^{\prime}, y^{\prime}\right)= & K_{x y l} \delta\left(x-x^{\prime}, y-y^{\prime}\right) \\
& \times \exp \left[-i \pi\left(\frac{x^{2}}{\lambda f_{x}}+\frac{y^{2}}{\lambda f_{y}}+\frac{x y}{\lambda f_{x y}}\right)\right],
\end{aligned}
$$

with the transformation matrix given by

$$
\mathbf{T}_{x y l}\left(f_{y}\right)=\left[\begin{array}{cccc}
0 & 1 & 0 & 0 \\
0 & 1 & 0 & 0 \\
\frac{-1}{\lambda f_{x}} & \frac{-1}{2 \lambda f_{x y}} & 1 & 0 \\
\frac{-1}{2 \lambda f_{x y}} & \frac{-1}{\lambda f_{y}} & 0 & 1
\end{array}\right] .
$$

The transformation-matrix approach is practical in the analysis of optical systems. First, if several systems are cascaded the overall system matrix can be found by multiplication of the corresponding transformation matrices. Second, the transformation matrix corresponds to the ray-matrix in optics. ${ }^{37}$ 
Third, the effect of the system on the Wigner distribution of the input function can be expressed in terms of this transformation matrix. This topic is discussed extensively in Refs. 35 and 38-41.

We already stated in Section 1 that the fractional Fourier transform belongs to the family of linear canonical transforms. So it is possible to calculate the transformation matrix for the fractional Fourier transform. Before finding the transformation matrix we modify the definition of the fractional Fourier transform. In some physical applications it is necessary to introduce input and output scale parameters. It is possible to modify our definition by inclusion of the scale parameters and also the additional phase factors that can occur at the output:

$$
\begin{aligned}
B_{a_{x}, a_{y}}\left(x, y ; x^{\prime}, y^{\prime}\right)= & A_{\phi_{x}} \exp \left(i \pi x^{2} p_{x}\right) \exp \left[i \pi \left(\frac{x^{2}}{s_{2}{ }^{2}} \cot \phi_{x}\right.\right. \\
& \left.\left.-\frac{2 x x^{\prime}}{s_{1} s_{2}} \csc \phi_{x}+\frac{x^{\prime 2}}{s_{1}{ }^{2}} \cot \phi_{x}\right)\right] \\
& \times A_{\phi_{y}} \exp \left(i \pi y^{2} p_{y}\right) \exp \left[i \pi \left(\frac{y^{2}}{s_{2}{ }^{2}} \cot \phi_{y}\right.\right. \\
& \left.\left.-\frac{2 y y^{\prime}}{s_{1} s_{2}} \csc \phi_{y}+\frac{y^{\prime 2}}{s_{1}{ }^{2}} \cot \phi_{y}\right)\right]
\end{aligned}
$$

In the definition in Eq. (38), $s_{1}$ stands for the input scale parameter and $s_{2}$ stands for the output scale parameter. With the phase factors $p_{x}, p_{y}$ and the scaling factors $s_{1}, s_{2}$ permitted, the transformation matrix of the fractional Fourier transform can be found as

$$
\mathbf{T} \equiv\left[\begin{array}{ll}
\mathbf{A} & \mathbf{B} \\
\mathbf{C} & \mathbf{D}
\end{array}\right],
$$

where

\section{Optical Implementation of Linear Canonical Transforms and Fractional Fourier Transforms by Use of Canonical Decompositions}

In our optical setups we try to control as many parameters as we can. Following is a list of parameters that we want to control:

- Order parameters $a_{x}$ and $a_{y}$ : The main objective of designing optical setups is to control the orders of the fractional Fourier transform. Control of the order parameters is our primary interest.

- Scale parameters $s_{1}$ and $s_{2}$ : It is desirable to specify both the input and the output scale parameters to provide practical setups.

- Additional phase factors $p_{x}$ and $p_{y}$ : In our designs we try to obtain $p_{x}=p_{y}=0$ to remove the additional phase factors at the output plane and observe the fractional Fourier transform on a flat surface.

In all the systems we analyze below we clearly indicate the parameters specified by the designer, the design parameters, and the uncontrollable outcomes, if any.

\section{A. One-Dimensional Systems}

\section{Canonical Decomposition Type 1}

The overall system matrix $\mathbf{T}$ of the system shown in Fig. 1 is

$$
\mathbf{T}=\mathbf{T}_{f}\left(d_{2}\right) \mathbf{T}_{x l}(f) \mathbf{T}_{f}\left(d_{1}\right) .
$$

Both the optical system depicted in Fig. 1 and the linear canonical transform have three parameters. Thus it is possible for one to find the system parameters uniquely by solving Eq. (44). The equations for

$$
\begin{aligned}
\mathbf{A} & =\left[\begin{array}{cc}
\frac{s_{2}}{s_{1}} \cos \phi_{x} & 0 \\
0 & \frac{s_{2}}{s_{1}} \cos \phi_{y}
\end{array}\right] \\
\mathbf{B} & =\left[\begin{array}{cc}
s_{1} s_{2} \sin \phi_{x} & 0 \\
0 & s_{1} s_{2} \sin \phi_{y}
\end{array}\right], \\
\mathbf{C} & =\left[\begin{array}{cc}
\frac{1}{s_{1} s_{2}}\left(p_{x} \cos \phi_{x}-\sin \phi_{x}\right) & 0 \\
0 & \frac{1}{s_{1} s_{2}}\left(p_{y} \cos \phi_{y}-\sin \phi_{y}\right)
\end{array}\right], \\
\mathbf{D} & =\left[\begin{array}{cc}
\frac{s_{1}}{s_{2}} \sin \phi_{x}\left(p_{x}+\cot \phi_{x}\right) & 0 \\
0 & \frac{s_{1}}{s_{2}} \sin \phi_{y}\left(p_{y}+\cot \phi_{y}\right)
\end{array}\right]
\end{aligned}
$$




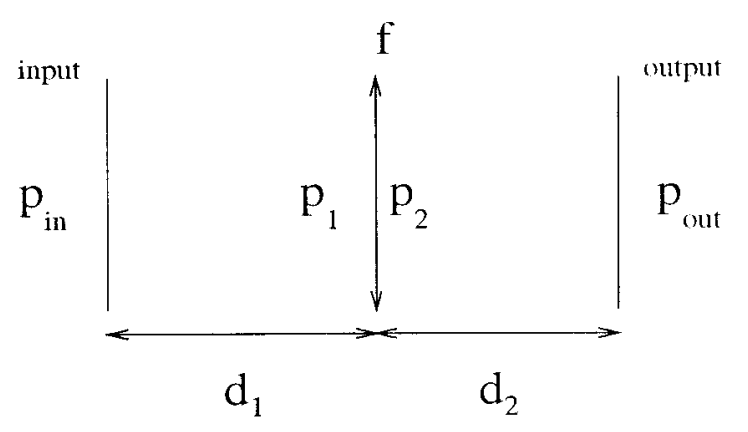

Fig. 1. Type 1 system that realizes the $1-D$ linear canonical transform.

$d_{1}, d_{2}$, and $f$ in terms of $\alpha, \beta$, and $\gamma$ are found as

$$
\begin{gathered}
d_{1}=\frac{\beta-\alpha}{\lambda\left(\beta^{2}-\gamma \alpha\right)}, \quad d_{2}=\frac{\beta-\gamma}{\lambda\left(\beta^{2}-\gamma \alpha\right)}, \\
f=\frac{\beta}{\lambda\left(\beta^{2}-\gamma \alpha\right)} .
\end{gathered}
$$

Since the fractional Fourier transform is a special form of linear canonical transforms, it is possible to implement a 1-D fractional Fourier transform of the desired order by use of this optical setup. The scale parameters $s_{1}$ and $s_{2}$ can be specified by the designer, and the additional phase factors $p_{x}$ and $p_{y}$ can be made equal to zero.

Letting $\alpha=\cot \phi / s_{2}{ }^{2}, \gamma=\cot \phi / s_{1}{ }^{2}$, and $\beta=\csc$ $\phi / s_{1} s_{2}$, one recovers the Lohmann type 1 system that performs the fractional Fourier transform. In this case the system parameters are found as

$$
\begin{gathered}
d_{1}=\frac{\left(s_{1} s_{2}-s_{1}{ }^{2} \cos \phi\right)}{\lambda \sin \phi}, \quad d_{2}=\frac{\left(s_{1} s_{2}-s_{2}{ }^{2} \cos \phi\right)}{\lambda \sin \phi}, \\
f=\frac{s_{1} s_{2}}{\lambda \sin \phi} .
\end{gathered}
$$

Since the additional phase factors are set to zero, they do not appear in Eqs. (46). However, if one wishes to set $p_{x}$ and $p_{y}$ to a value other than zero, it is again possible if we set $\alpha=p_{x} \cot \phi / s_{2}{ }^{2}$ and substitute it into Eqs. (45).

\section{Canonical Decomposition Type 2}

In this case, instead of one lens and two sections of free space, we have two lenses separated by a single section of free space, as shown in Fig. 2. Again, the parameters $d, f_{1}$, and $f_{2}$ are solved for in a manner similar to that of the type 1 decomposition:

$$
d=\frac{1}{\lambda \beta}, \quad f_{1}=\frac{1}{\lambda(\beta-\gamma)}, \quad f_{2}=\frac{1}{\lambda(\beta-\alpha)} .
$$

If $\alpha=\cot \phi / s_{2}{ }^{2}, \gamma=\cot \phi / s_{1}{ }^{2}$, and $\beta=\csc \phi / s_{1} s_{2}$ are substituted into Eqs. (47) the expressions for the fractional Fourier transform can be found. The designer can again specify the scale parameters, and there is no additional phase factor at the output. The system parameters are

$$
\begin{gathered}
d=\frac{s_{1} s_{2} \sin \phi}{\lambda}, \quad f_{1}=\frac{s_{1}{ }^{2} s_{2} \sin \phi}{s_{1}-s_{2} \cos \phi}, \\
f_{2}=\frac{s_{1} s_{2}{ }^{2} \sin \phi}{s_{2}-s_{1} \cos \phi} .
\end{gathered}
$$

Equations (45) and (47) give the expressions for the system parameters of type 1 and type 2 systems. But for some values of $\alpha, \beta$, and $\gamma$, the lengths of the free-space sections could turn out to be negative, which is not physically realizable. However, this constraint restricts the range of linear canonical transforms that can be realized with the suggested setups. In Section 3.C this problem is solved by use of an optical setup that simulates anamorphic and negative-valued sections of free space. This system is designed in such a way that its effect is equivalent to propagation in free space with different (and possibly negative) distances along the two dimensions.

\section{B. Optical Implementation of Two-Dimensional Linear} Canonical Transforms and Fractional Fourier Transforms

Here we present an elementary outcome that allows us to analyze 2-D systems as two 1-D systems. This result makes the analysis of 2-D systems remarkably easier. Let

$$
g(\mathbf{r})=\int_{-\infty}^{\infty} h\left(\mathbf{r}, \mathbf{r}^{\prime}\right) f\left(\mathbf{r}^{\prime}\right) \mathrm{d} \mathbf{r}^{\prime},
$$

where

$$
\mathbf{r}=\left[\begin{array}{ll}
x & y
\end{array}\right]^{T}, \quad \mathbf{r}^{\prime}=\left[\begin{array}{ll}
x^{\prime} & y^{\prime}
\end{array}\right]^{T} .
$$

If the kernel $h\left(\mathbf{r}, \mathbf{r}^{\prime}\right)$ is separable, i.e.,

$$
h\left(\mathbf{r}, \mathbf{r}^{\prime}\right)=h_{x}\left(x, x^{\prime}\right) h_{y}\left(y, y^{\prime}\right),
$$

then the response in the $x$ direction is the result of the 1-D transform

$$
g_{x}\left(x, y^{\prime}\right)=\int_{-\infty}^{\infty} h_{x}\left(x, x^{\prime}\right) f\left(x^{\prime}, y^{\prime}\right) \mathrm{d} x^{\prime}
$$

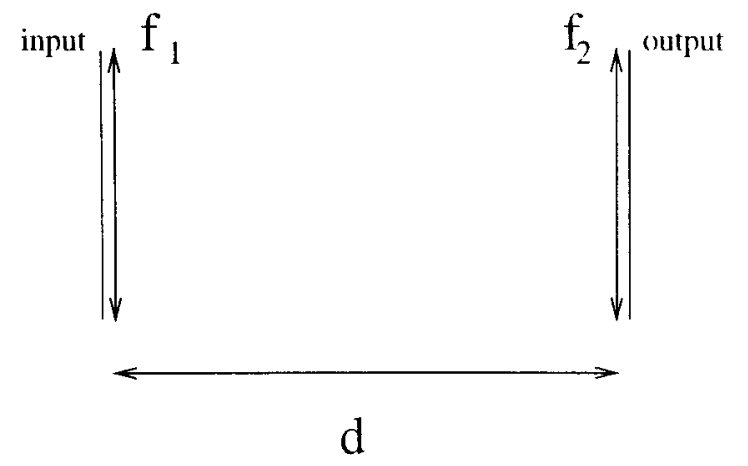

Fig. 2. Type 2 system that realizes the 1-D linear canonical transform. 
and is similar in the $y$ direction. Moreover, if the function is also separable, i.e.,

$$
f(\mathbf{r})=f_{x}(x) f_{y}(y)
$$

the overall response of the system is

$$
g(\mathbf{r})=g_{x}(x) g_{y}(y),
$$

where

$$
g_{x}(x)=\int_{-\infty}^{\infty} h_{x}\left(x, x^{\prime}\right) f_{x}\left(x^{\prime}\right) \mathrm{d} x^{\prime} .
$$

There is a similar expression for the $y$ direction. This result is easily verified by substitution of Eqs. (49) and (51) into Eq. (53):

$$
g(\mathbf{r})=\int_{-\infty}^{\infty} \int_{-\infty}^{\infty} h_{x}\left(x, x^{\prime}\right) h_{y}\left(y, y^{\prime}\right) f_{x}\left(x^{\prime}\right) f_{y}\left(y^{\prime}\right) \mathrm{d} x^{\prime} \mathrm{d} y^{\prime} .
$$

Rearranging the terms will give us the desired outcome.

The result above has a nice interpretation in optics, which makes the analysis of 2-D systems easier. For example, to design an optical setup that realizes imaging in the $x$ direction and the Fourier transform in the $y$ direction, one can design two 1-D systems that realize the given transformations. When these two systems are merged, the overall effect of the system is imaging in the $x$ direction and Fourier transformation in the $y$ direction. Similarly, if we can find a system that realizes the fractional Fourier transform with the order $a_{x}$ in the $x$ direction and another system that realizes the fractional Fourier transform with the order $a_{y}$ in the $y$ direction, then these two optical setups together will implement the 2-D fractional Fourier transform. So the problem of designing a 2-D fractional Fourier transformer reduces to the problem of designing two 1-D fractional Fourier transformers.

\section{Canonical Decomposition Type 1}

According to the above result, the $x$ and $y$ directions can be considered independently of each other, since the kernel given in Eqs. (29) is separable. Hence if two optical setups that achieve 1-D linear canonical transforms are put together, one can implement the desired 2-D fractional Fourier transform. The suggested optical system is shown in Fig. 3.

The parameters of the type 1 system are as follows:

$$
\begin{aligned}
d_{1 x} & =\frac{\beta_{x}-\alpha_{x}}{\lambda\left(\beta_{x}^{2}-\gamma_{x} \alpha_{x}\right)}, \quad d_{2 x}=\frac{\beta_{x}-\gamma_{x}}{\lambda\left(\beta_{x}^{2}-\gamma_{x} \alpha_{x}\right)}, \\
f_{x} & =\frac{\beta_{x}}{\lambda\left(\beta_{x}^{2}-\gamma_{x} \alpha_{x}\right)}, \\
d_{1 y} & =\frac{\beta_{y}-\alpha_{y}}{\lambda\left(\beta_{y}^{2}-\gamma_{y} \alpha_{y}\right)}, \\
f_{y} & =\frac{\beta_{y}}{\lambda\left(\beta_{y}{ }^{2}-\gamma_{y} \alpha_{y}\right)} .
\end{aligned}
$$

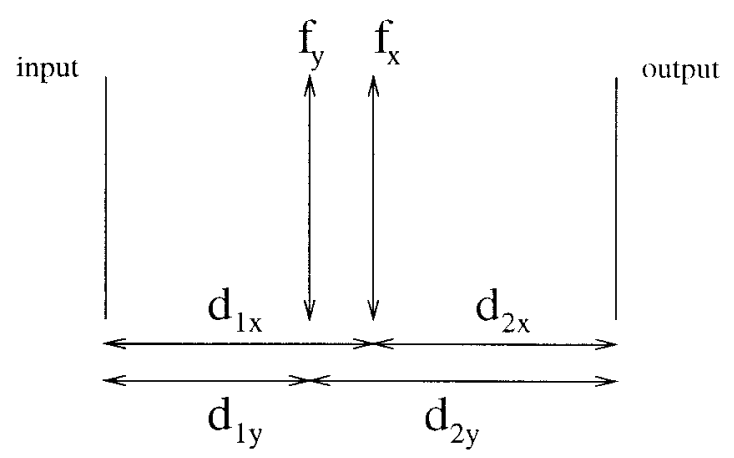

Fig. 3. Type 1 system that realizes 2-D linear canonical transforms.

It was discussed in Subsection 2.B that a 2-D fractional Fourier transform is indeed a special linear canonical system with the parameters

$\begin{array}{llr}\alpha_{x}=\cot \phi_{x} / s_{2}{ }^{2}, & \gamma_{x}=\cot \phi_{x} / s_{1}{ }^{2}, & \beta_{x}=\csc \phi_{x} / s_{1} s_{2}, \\ \alpha_{y}=\cot \phi_{y} / s_{2}{ }^{2}, & \gamma_{y}=\cot \phi_{y} / s_{1}{ }^{2}, & \beta_{y}=\csc \phi_{y} / s_{1} s_{2} .\end{array}$

When Eqs. (56) and (57) are substituted into Eqs. (54) and (55), the parameters of the fractional Fourier transforming optical system can be found. Even though the analysis is carried out by use of the independence of the $x$ and $y$ directions, the total length of the optical system is fixed. Thus the condition $d_{1 x}+$ $d_{2 x}=d_{x}=d_{1 y}+d_{2 y}=d_{y}$ should always be satisfied. The other constraint to be satisfied is the positivity of the lengths of the free-space sections: $d_{1 x}, d_{1 y}, d_{2 x}$, and $d_{2 y}$ should always be positive. These two constraints restrict the set of linear canonical transforms that can be implemented. The solution to this problem is to simulate anamorphic sections of free space. Such simulation provides us with the propagation of $d_{x}$ in the $x$ direction and of $d_{y}$ in the $y$ direction, where $d_{x}$ and $d_{y}$ can take negative values. This problem is solved in Subsection 3.B.

\section{Canonical Decomposition Type 2}

Two type 2 systems can also perform the desired 2-D linear canonical transforms. The parameters of the type 2 system are as follows:

$$
\begin{array}{lll}
d_{x}=\frac{1}{\lambda \beta_{x}}, & f_{1 x}=\frac{1}{\lambda\left(\beta_{x}-\gamma_{x}\right)}, & f_{2 x}=\frac{1}{\lambda\left(\beta_{x}-\alpha_{x}\right)}, \\
d_{y}=\frac{1}{\lambda \beta_{y}}, & f_{1 y}=\frac{1}{\lambda\left(\beta_{y}-\gamma_{y}\right)}, & f_{2 y}=\frac{1}{\lambda\left(\beta_{y}-\alpha_{y}\right)} .
\end{array}
$$

If Eqs. (56) and (57) are substituted into Eqs. (58) and (59) we have the parameters for the fractional Fourier transform, which is indeed a linear canonical transform.

The optical setup shown in Fig. 4, with the param- 


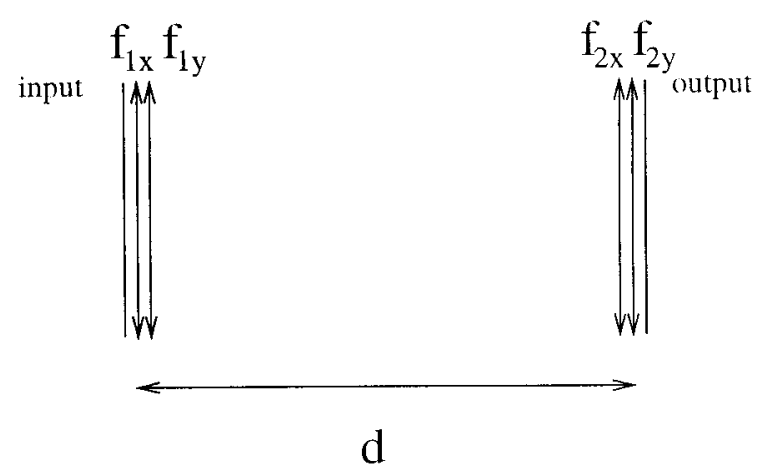

Fig. 4. Type 2 system that realizes the 2-D linear canonical transform.

eters given in Eqs. (58) and (59), implement the 2-D linear canonical transform. In this optical setup the constraint becomes $d_{x}=d_{y}=d$, which is even more restrictive than that for type 1 systems. The terms $d_{x}$ and $d_{y}$ can again be negative. To overcome these difficulties, we try to design an optical setup that simulates anamorphic sections of free space.

\section{Simulation of Anamorphic Sections of Free Space}

While designing optical setups that implement 1-D linear canonical transforms we treat the lengths of the free-space sections as free parameters. But some linear canonical transforms specified by the parameters $\alpha, \gamma$, and $\beta$ might require the use of freespace sections with negative length. This problem is again encountered in the optical setups that achieve 2-D linear canonical transforms. Besides, the 2-D optical systems require different propagation distances in the $x$ and the $y$ directions. To implement all possible 1-D and 2-D linear canonical transforms, we design an optical system that simulates the desired free space suitable for our purposes. The optical system shown in Fig. 5, which comprises a Fourier block, an anamorphic lens, and an inverse Fourier block, simulates 2-D free space with the propagation distance $d_{x}$ in the $x$ direction and $d_{y}$ in the $y$ direction. We call the optical system shown in Fig. 5 an anamorphic free-space system. When the analysis of the system illustrated in Fig. 5 is carried out, the relation between the input light distribution $f(x$, $y$ ) and the output light distribution $g(x, y)$ is given as

$$
\begin{aligned}
g(x, y)= & C \int_{-\infty}^{\infty} \int_{-\infty}^{\infty} \exp \left[i \pi\left(x-x^{\prime}\right)^{2} / \lambda d_{x}\right. \\
& \left.+\left(y-y^{\prime}\right)^{2} / \lambda d_{y}\right] f\left(x^{\prime}, y^{\prime}\right) \mathrm{d} x^{\prime} \mathrm{d} y^{\prime},
\end{aligned}
$$

where

$$
d_{x}=\frac{s^{4}}{\lambda^{2} f_{x}}, \quad d_{y}=\frac{s^{4}}{\lambda^{2} f_{y}},
$$

and $s$ is the scale of the Fourier and the inverse Fourier blocks. The terms $f_{x}$ and $f_{y}$ can take any real value, including negative ones. Thus it is possible to obtain any combination of $d_{x}$ and $d_{y}$ by use of the

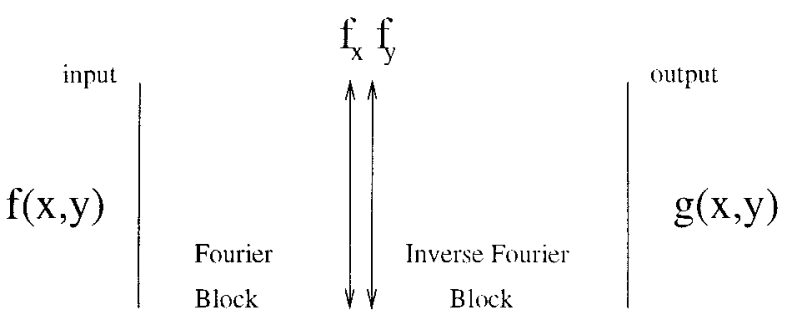

Fig. 5. Optical system that simulates anamorphic free-space propagation.

optical setup depicted in Fig. 5. The anamorphic lens, which is used to control $d_{x}$ and $d_{y}$, can be composed of two orthogonally situated cylindrical thin lenses with different focal lengths. The Fourier block and the inverse Fourier block are $2 f$ systems with a spherical lens between two sections of free space. Thus a section of free space uses two cylindrical and two spherical lenses.

The system represented in Fig. 5 simulates 2-D anamorphic free space. The same configuration is again valid for the 1-D case. When only one lens is used with the 1-D Fourier and inverse Fourier blocks it is possible to simulate propagation with negative distances. When the free-space sections in the type 1 and type 2 systems are replaced with the optical setup of Fig. 5, optical implementation of all separable linear canonical transforms can be realized.

Both type 1 and type 2 systems can be used to implement all combinations of orders when the freespace sections are replaced with sections of anamorphic free space. We have no residual phase factors at the output. Also, the scale parameters can be specified by the designer. Thus by use of type 1 and type 2 systems all combinations of the orders $a_{x}$ and $a_{y}$ can be implemented with full control of the scale parameters $s_{1}, s_{2}$ and the phase factors $p_{x}, p_{y}$.

\section{Other Optical Implementations of Two-Dimensional Fractional Fourier Transforms}

In Section 3 we presented a method for implementing the fractional Fourier transform optically. All combinations of $a_{x}$ and $a_{y}$ can be implemented with the proposed setups; however, both systems use six cylindrical lenses. In this section we consider simpler optical systems having fewer lenses and try to see the limitations of these systems. We do not attempt to exhaust all possibilities but offer several systems that we believe may be useful. Since the problem is solved in the $x$ and $y$ directions independently, one lens is not adequate for controlling both directions. So the simplest setup that we consider has two cylindrical lenses.

\section{A. Two-Lens Systems}

\section{Setup with Six Design Parameters}

This setup has the following parameters:

- Parameters specified by the designer: $\phi_{x}, \phi_{y}$, $s_{1}, s_{2}, p_{x}, p_{y}$. 
- Design parameters: $f_{x}, f_{y}, d_{1 x}, d_{1 y}, d_{2 x}, d_{2 y}$.

- Uncontrollable outcomes: None.

The optical setup shown in Fig. 5 has six design parameters, and we also want to specify six parameters here. It is possible to solve the design parameters in terms of the desired parameters determined by the designer. However, to produce a realizable setup, we should satisfy the following constraints:

- The total length of the system should be the same in both directions: $d_{1 x}+d_{2 x}=d_{1 y}+d_{2 y}$.

- The lengths of all free-space sections should be positive: $d_{1 x} \geq 0, d_{1 y} \geq 0, d_{2 x} \geq 0$, and $d_{2 y} \geq 0$.

These constraints are too restrictive, and the range of orders $a_{x}$ and $a_{y}$ that can be implemented is small. Thus we have to reduce the number of parameters that we want to control. This method is considered in Subsection 4.A.2.

\section{Setup with Fewer Parameters}

This system has the following parameters:

- Parameters specified by the designer: $\phi_{x}, \phi_{y}$, $s_{1}, s_{2}$.

- Design parameters: $f_{x}, f_{y}, d_{1 x}, d_{1 y}, d_{2 x}, d_{2 y}$.

- Uncontrollable outcomes: $p_{x}, p_{y}$.

In this design both the orders and the scale parameters can be specified. For the given parameters $\phi_{x}$, $\phi_{y}$ and $s_{1}, s_{2}$, the design parameters are

$$
\begin{aligned}
& d_{1 x}=d_{1 y}=d_{1}=\frac{s_{1}{ }^{2}\left(\sin \phi_{y}-\sin \phi_{x}\right)}{\lambda\left(\cos \phi_{y}-\cos \phi_{x}\right)}, \\
& d_{2 x}=d_{2 y}=d_{2}=\frac{s_{1} s_{2} \sin \left(\phi_{x}-\phi_{y}\right)}{\lambda\left(\cos \phi_{y}-\cos \phi_{x}\right)}, \\
& f_{x}=\frac{s_{1}{ }^{2} s_{2} \sin \left(\phi_{x}-\phi_{y}\right)}{\lambda\left(s_{1}-s_{2} \cos \phi_{x}\right)\left(\cos \phi_{y}-\cos \phi_{x}\right)}, \\
& f_{y}=\frac{s_{1}{ }^{2} s_{2} \sin \left(\phi_{x}-\phi_{y}\right)}{\lambda\left(s_{1}-s_{2} \cos \phi_{y}\right)\left(\cos \phi_{y}-\cos \phi_{x}\right)},
\end{aligned}
$$

and the phase factors at the output plane turn out to be

$$
\begin{aligned}
& p_{x}=\frac{\left\{s_{2}\left(\cos \phi_{y}-\cos \phi_{x}\right)+s_{1}\left[1-\cos \left(\phi_{y}-\phi_{x}\right)\right]\right\}}{s_{1} s_{2}{ }^{2} \sin \left(\phi_{x}-\phi_{y}\right)}, \\
& p_{y}=\frac{\left\{s_{2}\left(\cos \phi_{y}-\cos \phi_{x}\right)+s_{1}\left[\cos \left(\phi_{y}-\phi_{x}\right)-1\right]\right\}}{s_{1} s_{2}{ }^{2} \sin \left(\phi_{x}-\phi_{y}\right)} .
\end{aligned}
$$

In this optical setup $d_{1}$ and $d_{2}$ should always be positive (see Fig. 6). But for some values of $\phi_{x}, \phi_{y}, s_{1}$, and $s_{2}$, the values of $d_{1}$ and $d_{2}$ may turn out to be negative. In such cases we would have to deal with virtual objects, virtual images, or both. This would require the use of additional lenses. To avoid this we must require that $d_{1}$ and $d_{2}$ be positive. This condition will restrict the ranges of $a_{x}$ and $a_{y}$ that can

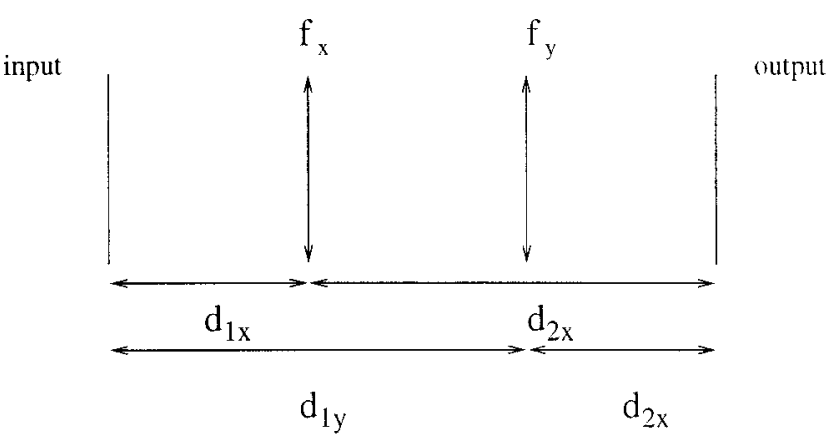

Fig. 6. Optical setup with two cylindrical lenses and three sections of free space.

be realized. These ranges can be maximized if the $x$ or the $y$ axis is flipped. For instance, if the given values of $d_{1 x}, d_{2 x}, d_{1 y}$, and $d_{2 y}$ make $s_{1}$ negative for $\phi_{x}=60$ and $\phi_{y}=30$, we flip one of the axes. This transform is equivalent to the fractional Fourier transform, with $\phi_{x}=60$ and $\phi_{y}=210$, followed by a flip of the $y$ axis or to the fractional Fourier transform, with $\phi_{x}=240$ and $\phi_{y}=30$, followed by a flip of the $x$ axis. (This is because a second-order transform corresponds to a flip of the coordinate axis.) To implement some orders we must flip both axes. Figure 7 shows the necessary flip(s) required to realize different combinations of orders.

The above system allows us to specify the orders and the scale parameters. However, the phase factors are arbitrary and out of our control. We should examine four-lens systems if we wish to control the orders, the scale parameters, and the phase factors at the same time.

\section{B. Four-Lens Systems}

\section{Setup with Adjustable Length}

We continue our discussion with the setup shown in Fig. 8:

- Parameters specified by the designer: $\phi_{x}, \phi_{y}$, $s_{1}, s_{2}, p_{x}=p_{y}=0$.

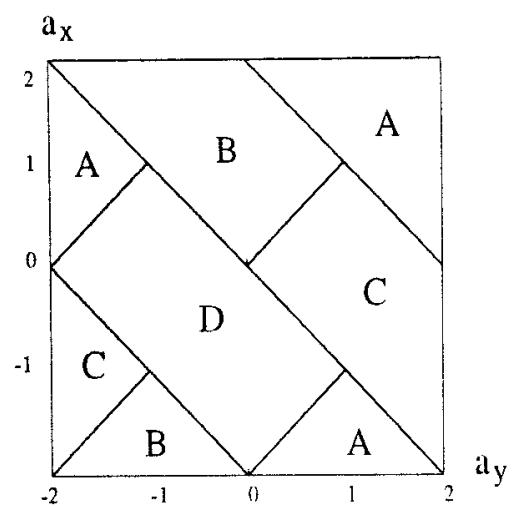

Fig. 7. Sections A: Neither axis flipped. Sections B: $x$ axis flipped. Sections C: $y$ axis flipped. Section D: Both axes flipped. 


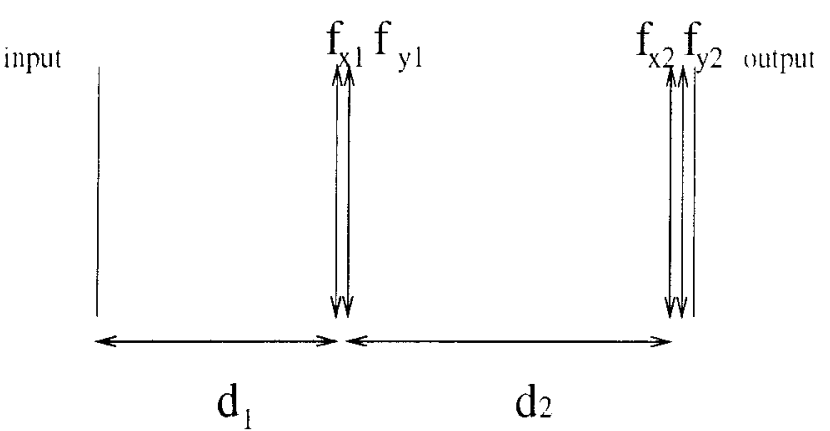

Fig. 8. Optical setup with four cylindrical lenses and two sections of free space.

- Design parameters: $d_{1}, d_{2}, f_{x 1}, f_{y 1}, f_{x 2}, f_{y 2}$.

- Uncontrollable outcomes: None.

In this configuration we use the optical setup depicted in Fig. 8. In our design with two lenses (Subsection 4.A), we managed to design an optical setup that implements the 2-D fractional Fourier transform with the orders and scale parameters we desired. However, additional phase factors at the output plane turned out to be arbitrary. If two cylindrical lenses are added to the output plane of the two-lens system, it is possible to remove the additional phase factor at the output. In the optical setup of Fig. 8, $d_{1}, d_{2}, f_{x 1}$, and $f_{x 2}$ have the same expressions as those for the two-lens system. Thus Fig. 7 is again valid and shows the necessary flips:

$$
\begin{aligned}
& d_{1 x}=d_{1 y}=d_{1}=\frac{s_{1}^{2}\left(\sin \phi_{y}-\sin \phi_{x}\right)}{\lambda\left(\cos \phi_{y}-\cos \phi_{x}\right)} \\
& d_{2 x}=d_{2 y}=d_{2}=\frac{s_{1} s_{2} \sin \left(\phi_{x}-\phi_{y}\right)}{\lambda\left(\cos \phi_{y}-\cos \phi_{x}\right)}, \\
& f_{x 1}=\frac{s_{1}{ }^{2} s_{2} \sin \left(\phi_{x}-\phi_{y}\right)}{\lambda\left(s_{1}-s_{2} \cos \phi_{x}\right)\left(\cos \phi_{y}-\cos \phi_{x}\right)}, \\
& f_{y 1}=\frac{s_{1}{ }^{2} s_{2} \sin \left(\phi_{x}-\phi_{y}\right)}{\lambda\left(s_{1}-s_{2} \cos \phi_{y}\right)\left(\cos \phi_{y}-\cos \phi_{x}\right)}, \\
& f_{x 2}=\frac{s_{1} s_{2}{ }^{2} \sin \left(\phi_{x}-\phi_{y}\right)}{\lambda\left\{s_{2}\left(\cos \phi_{y}-\cos \phi_{x}\right)+s_{1}\left[1-\cos \left(\phi_{y}-\phi_{x}\right)\right]\right\}}, \\
& f_{y 2}=\frac{s_{1} s_{2}{ }^{2} \sin \left(\phi_{x}-\phi_{y}\right)}{\lambda\left\{s_{2}\left(\cos \phi_{y}-\cos \phi_{x}\right)+s_{1}\left[\cos \left(\phi_{y}-\phi_{x}\right)-1\right]\right\}} .
\end{aligned}
$$

This optical setup implements the 2-D fractional Fourier transform with the desired orders, scale parameters, and phase factors.

\section{Setup with Fixed Length}

This system has the following parameters:

- Parameters specified by the designer: $\phi_{x}, \phi_{y}$, $s_{1}, s_{2}, d_{1}, d_{2}, d_{3}$.

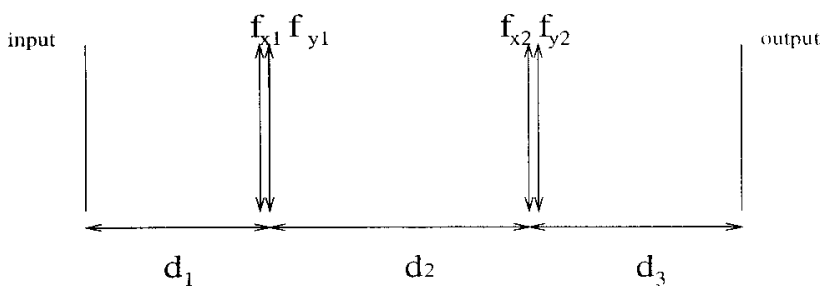

Fig. 9. Optical setup with four cylindrical lenses and three sections of free space.

- Design parameters: $f_{x 1}, f_{y 1}, f_{x 2}, f_{y 2}$.

- Uncontrollable outcomes: $p_{x}, p_{y}$.

For practical purposes one may want to use a fixed system in which the lengths of all free-space sections are fixed. For example, Ref. 26 reports that the 2-D fractional Fourier transform is implemented by use of cylindrical lenses with dynamically adjustable focal lengths in a fixed system. Both the locations of the lenses and the total length of the system are fixed. The only design parameters are the focal lengths of the lenses, which can be changed dynamically.

Here we add one more section of free space to the system shown in Fig. 8 and obtain the setup shown in Fig. 9. This fixed system exerts no control over the phase factors, while the orders and the scale parameters can be specified by the designer. The parameters are

$$
\begin{aligned}
& f_{x 1}=\frac{s_{1} s_{2} d_{2} \sin \phi_{x} / \lambda-\left(s_{2} / s_{1}\right) d_{1} d_{2} \cos \phi_{x}}{\left(s_{2} / s_{1}\right)\left(d_{1}+d_{2}\right) \cos \phi_{x}-s_{1} s_{2} \sin \phi_{x} / \lambda+d_{3}}, \\
& f_{y 1}=\frac{s_{1} s_{2} d_{2} \sin \phi_{y} / \lambda-\left(s_{2} / s_{1}\right) d_{1} d_{2} \cos \phi_{y}}{\left(s_{2} / s_{1}\right)\left(d_{1}+d_{2}\right) \cos \phi_{y}-s_{1} s_{2} \sin \phi_{y} / \lambda+d_{3}}, \\
& f_{x 2}=\frac{d_{2} d_{3}}{\left(s_{2} / s_{1}\right) d_{1} \cos \phi_{x}-s_{1} s_{2} \sin \phi_{x} / \lambda+d_{2}+d_{3}}, \\
& f_{y 2}=\frac{d_{2} d_{3}}{\left(s_{2} / s_{1}\right) d_{1} \cos \phi_{y}-s_{1} s_{2} \sin \phi_{y} / \lambda+d_{2}+d_{3}},
\end{aligned}
$$

and the additional phase factors turn out to be

$$
\begin{aligned}
& p_{x}=-\cos \phi_{x}+\frac{s_{2}}{s_{1} \sin \phi_{x}}\left(1-\frac{d_{1}}{f_{x 1}}-\frac{d_{1}}{f_{x 2}}-\frac{d_{2}}{f_{x 2}}+\frac{d_{1} d_{2}}{f_{x 1} f_{x 2}}\right), \\
& p_{y}=-\cos \phi_{y}+\frac{s_{2}}{s_{1} \sin \phi_{y}}\left(1-\frac{d_{1}}{f_{y 1}}-\frac{d_{1}}{f_{y 2}}-\frac{d_{2}}{f_{y 2}}+\frac{d_{1} d_{2}}{f_{y 1} f_{y 2}}\right) .
\end{aligned}
$$

This optical setup can be used to realize all combinations of $a_{x}$ and $a_{y}$; however, there are additional uncontrollable phase factors observed at the output plane.

\section{Six-Lens System}

This system has the following parameters: 


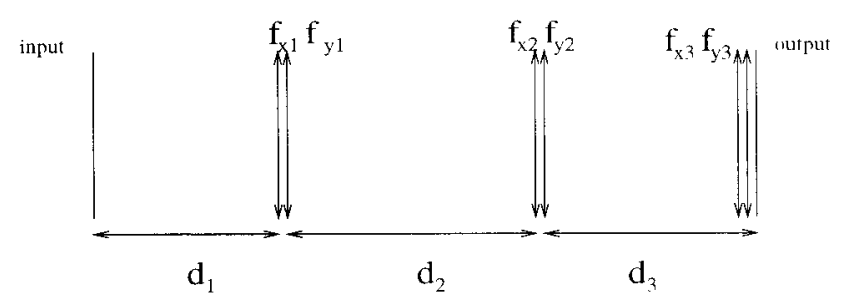

Fig. 10. Optical system with six lenses and three sections of free space.

- Parameters specified by the designer: $\phi_{x}, \phi_{y}$, $s_{1}, s_{2}, d_{1}, d_{2}, d_{3}, p_{x}=p_{y}=0$.

- Design parameters: $f_{x 1}, f_{y 1}, f_{x 2}, f_{y 2}$.

- Uncontrollable outcomes: None.

The modified type 1 and type 2 systems use six cylindrical lenses. However, the lengths of the freespace sections are not fixed. For practical purposes, as we mentioned in Subsection 4.B.2, one may want to use a fixed system. To have control over all the parameters, we require a six-lens system. The design that we made using the four-lens fixed system has two uncontrollable outcomes: $p_{x}$ and $p_{y}$. If two cylindrical lenses are added to the output plane, full control over all parameters is achieved.

The system parameters $f_{x 1}, f_{y 1}, f_{2 x}$, and $f_{y 2}$ are the same as those for the four-lens fixed system. The focal lengths of the additional lenses are

$$
\begin{aligned}
& f_{x 3}=\frac{1}{\lambda p_{x}}, \\
& f_{y 3}=\frac{1}{\lambda p_{y}} .
\end{aligned}
$$

Thus the fixed optical system shown in Fig. 10 can be used to implement the desired fractional Fourier transform.

\section{Conclusion}

We have presented a systematic treatment of the 2-D fractional Fourier transform and its optical implementation. We have provided design equations for a system composed of four cylindrical lenses, in which the user can specify the transform orders in two dimensions, the input and output scale parameters, and the residual phase factors appearing at the output plane. Many other systems with fewer or more lenses and with less or more flexibility when specifying parameters have also been discussed.

The systems we discussed in Section 3 are good for realizing arbitrary linear canonical transforms. Such transforms are a more general class of transforms than the fractional Fourier transforms.

\section{References}

1. H. M. Ozaktas and D. Mendlovic, "Fourier transforms of fractional order and their optical interpretation," Opt. Commun. 101, 163-169 (1993).

2. D. Mendlovic and H. M. Ozaktas, "Fractional Fourier trans- forms and their optical implementation I," J. Opt. Soc. Am. A 10, 1875-1881 (1993).

3. H. M. Ozaktas and D. Mendlovic, "Fractional Fourier transforms and their optical implementation II," J. Opt. Soc. Am. A 10, 2522-2531 (1993).

4. A. W. Lohmann, "Image rotation, Wigner rotation, and the fractional order Fourier transform," J. Opt. Soc. Am. A 10, 2181-2186 (1993).

5. D. Mendlovic, H. M. Ozaktas, and A. W. Lohmann, "Gradedindex fibers, Wigner-distribution functions, and the fractional Fourier transform," Appl. Opt. 33, 6188-6193 (1994).

6. H. M. Ozaktas and D. Mendlovic, "Fractional Fourier optics," J. Opt. Soc. Am. A 12, 743-751 (1995).

7. P. Pellat-Finet, "Fresnel diffraction and the fractional-order Fourier transform," Opt. Lett. 19, 1388-1390 (1994).

8. P. Pellat-Finet and G. Bonnet, "Fractional order Fourier transform and Fourier optics," Opt. Commun. 111, 141-154 (1994).

9. D. Mendlovic, H. M. Ozaktas, and A. W. Lohmann, "Fractional correlation,” Appl. Opt. 34, 303-309 (1995).

10. L. M. Bernardo and O. D. D. Soares, "Fractional Fourier transforms and imaging," J. Opt. Soc. Am. A 11, 2622-2626 (1994).

11. L. M. Bernardo and O. D. D. Soares, "Fractional Fourier transforms and optical systems," Opt. Commun. 110, 517-522 (1994).

12. H. M. Ozaktas and D. Mendlovic, "Fractional Fourier transform as a tool for analyzing beam propagation and spherical mirror resonators," Opt. Lett. 19, 1678-1680 (1994).

13. T. Alieva, V. Lopez, F. Agullo-Lopez, and L. B. Almedia, "The fractional Fourier transform in optical propagation problems," J. Mod. Opt. 41, 1037-1044 (1994).

14. M. A. Kutay, H. M. Ozaktas, L. Onural, and O. Arikan, "Optimal filtering in fractional Fourier domains," in Proceedings of the 1995 IEEE International Conference on Acoustics, Speech, and Signal Processing (Institute of Electrical and Electronics Engineers, New York, 1995), Vol. 12, pp. 937-940.

15. M. A. Kutay, H. M. Ozaktas, O. Arikan, and L. Onural, "Optimal filtering in fractional Fourier domains," IEEE Trans. Signal Process. 45, 1129-1143 (1997).

16. H. M. Ozaktas, O. Arikan, M. A. Kutay, and G. Bozdagi, "Digital computation of the fractional Fourier transform," IEEE Trans. Signal Process. 44, 2141-2150 (1996).

17. R. G. Dorsch, A. W. Lohmann, Y. Bitran, D. Mendlovic, and H. M. Ozaktas, "Chirp filtering in the fractional Fourier domain,” Appl. Opt. 33, 7599-7602 (1994).

18. D. Mendlovic, Z. Zalevsky, A. W. Lohmann, and R. G. Dorsch, "Signal spatial-filtering using the localized fractional Fourier transform," Opt. Commun. 126, 14-18 (1996).

19. A. W. Lohmann, Z. Zalevsky, and D. Mendlovic, "Synthesis of pattern recognition filters for fractional Fourier processing," Opt. Commun. 128, 199-204 (1996).

20. Z. Zalevsky and D. Mendlovic, "Fractional Wiener filter," Appl. Opt. 35, 3930-3936 (1996).

21. D. F. McAlister, M. Beck, L. Clarke, A. Mayer, and M. G. Raymer, "Optical phase retrieval by phase-space tomography and fractional-order Fourier transforms," Opt. Lett. 20, 11811183 (1995).

22. J. Garcia, D. Mendlovic, Z. Zalevsky, and A. Lohmann, "Spacevariant simultaneous detection of several objects by the use of multiple anamorphic fractional-Fourier-transform filters," Appl. Opt. 35, 3945-3952 (1996).

23. B. Barshan, M. A. Kutay, and H. M. Ozaktas, "Optimal filtering with linear canonical transformations," Opt. Commun. 135, 32-36 (1997).

24. H. M. Ozaktas, "Repeated fractional Fourier domain filtering is equivalent to repeated time and frequency domain filtering," Signal Process. 54, 81-84 (1996).

25. H. M. Ozaktas and D. Mendlovic, "Every Fourier optical sys- 
tem is equivalent to consecutive fractional Fourier domain filtering," Signal Process. 54, 81-84 (1996).

26. M. F. Erden, H. M. Ozaktas, A. Sahin, and D. Mendlovic, "Design of dynamically adjustable fractional Fourier transformer," Opt. Commun. 136, 52-60 (1997).

27. D. Mendlovic, Y. Bitran, C. Ferreira, J. Garcia, and H. M. Ozaktas, "Anamorphic fractional Fourier transformingoptical implementation and applications," Appl. Opt. 34, 74517456 (1995).

28. A. Sahin, H. M. Ozaktas, and D. Mendlovic, "Optical implementation of the two-dimensional fractional Fourier transform with different orders in the two dimensions," Opt. Commun. 120, 134-138, (1995).

29. A. Sahin, "Two-dimensional fractional Fourier transform and its optical implementation," M.S. thesis (Bilkent University, Ankara, Turkey, 1996).

30. Y. B. Karasik, "Expression of the kernel of a fractional Fourier transform in elementary functions," Opt. Lett. 19, 769-770 (1994).

31. L. B. Almeida, "The fractional Fourier transform and timefrequency representations," IEEE Trans. Signal Process. 42, 3084-3091 (1994).

32. V. Namias, "The fractional order Fourier transform and its application to quantum mechanics," J. Inst. Math. Its Appl. 25, 241-265 (1987).
33. A. C. McBride and F. H. Kerr, "On Namias's fractional Fourier transform," IMA J. Appl. Math. 39, 159-175.

34. H. M. Ozaktas, B. Barshan, D. Mendlovic, and L. Onural, "Convolution, filtering, and multiplexing in fractional Fourier domains and their relation to chirp and wavelet transforms," J. Opt. Soc. Am. A 11, 547-559 (1994).

35. M. J. Bastiaans, "Wigner distribution function and its application to first-order optics," J. Opt. Soc. Am. A 69, 1710-1716 (1979).

36. K. B. Wolf, "Construction and properties of canonical transforms," in Integral Transforms in Science and Engineering (Plenum, New York, 1979), Chap. 9.

37. B. E. A. Saleh and M. C. Teich, Fundamentals of Photonics (Wiley, New York, 1991).

38. M. J. Bastiaans, "The Wigner distribution applied to optical signals and systems," Opt. Commun. 25, 26-30 (1978).

39. M. J. Bastiaans, "The Wigner distribution function and Hamilton's characteristics of a geometric-optical system," Opt. Commun. 30, 321-326 (1979).

40. M. J. Bastiaans, "Propagation laws for the second-order moments of the Wigner distribution in first-order optical systems," Optik (Stuttgart) 82, 173-181 (1989).

41. M. J. Bastiaans, "Second-order moments of the Wigner distribution function in first-order optical systems," Optik (Stuttgart) 88, 163-168 (1991). 